\title{
When does stress help or harm? The effects of stress controllability and subjective stress response on Stroop performance
}

\author{
Roselinde K. Henderson ${ }^{1}$ *, Hannah R. Snyder ${ }^{1}$, Tina Gupta ${ }^{1}$ and Marie T. Banich ${ }^{1,2}$ \\ ${ }^{1}$ Department of Psychology and Neuroscience, University of Colorado at Boulder, Boulder, CO, USA \\ 2 Institute of Cognitive Science, University of Colorado at Boulder, Boulder, CO, USA
}

Edited by:

Florin Dolcos, University of Illinois Urbana-Champaign, USA

\section{Reviewed by:}

Oliver T. Wolf, Ruhr University

Bochum, Germany

Shaozheng Qin, Stanford University, USA

Marloes J. A. G. Henckens, Donders Institute for Brain, Cognition and

Behaviour, Radboud University

Nijmegen, Netherlands

${ }^{*}$ Correspondence:

Roselinde K. Henderson, Department of Psychology and Neuroscience,

University of Colorado at Boulder, 345 USB, Boulder, CO 80309, USA.

e-mail: roselinde.kaiser@colorado.edu
The ability to engage in goal-directed behavior despite exposure to stress is critical to resilience. Questions of how stress can impair or improve behavioral functioning are important in diverse settings, from athletic competitions to academic testing. Previous research suggests that controllability is a key factor in the impact of stress on behavior: learning how to control stressors buffers people from the negative effects of stress on subsequent cognitively demanding tasks. In addition, research suggests that the impact of stress on cognitive functioning depends on an individual's response to stressors: moderate responses to stress can lead to improved performance while extreme (high or low) responses can lead to impaired performance. The present studies tested the hypothesis that (1) learning to behaviorally control stressors leads to improved performance on a test of general executive functioning, the color-word Stroop, and that (2) this improvement emerges specifically for people who report moderate (subjective) responses to stress. Experiment 1: Stroop performance, measured before and after a stress manipulation, was compared across groups of undergraduate participants $(n=109)$. People who learned to control a noise stressor and received accurate performance feedback demonstrated reduced Stroop interference compared with people exposed to uncontrollable noise stress and feedback indicating an exaggerated rate of failure. In the group who learned behavioral control, those who reported moderate levels of stress showed the greatest reduction in Stroop interference. In contrast, in the group exposed to uncontrollable events, self-reported stress failed to predict performance. Experiment 2: In a second sample $(n=90)$, we specifically investigated the role of controllability by keeping the rate of failure feedback constant across groups. In the group who learned behavioral control, those who reported moderate levels of stress showed the greatest Stroop improvement. Once again, this pattern was not demonstrated in the group exposed to uncontrollable events. These results suggest that stress controllability and subjective response interact to affect high-level cognitive abilities. Specifically, exposure to moderate, controllable stress benefits performance, but exposure to uncontrollable stress or having a more extreme response to stress tends to harm performance. These findings may provide insights on how to leverage the beneficial effects of stress in a range of settings.

Keywords: stress, executive function, controllability, contingency

\section{INTRODUCTION}

Stress is part of life. Pursuing goals despite exposure to stressors, or better yet, showing enhanced functioning in response to stress, are abilities that are fundamental to survival and resilience (Maier and Watkins, 2010). For a broad range of daily goals, it is critical to know what type of stress can help or harm behavioral functioning. Musical concerts, athletic competitions, and academic testing are all settings in which stress may either impair performance or fuel pursuit of goals. To perform optimally, healthy humans must expose themselves to the types of stress that promote the most enhanced functioning possible.
The effects of stress on cognitive functions, specifically, may mediate the helpful and harmful effects of stress in complex domains such as those described above. Stress research with humans has yielded evidence for both positive and negative effects of stress and stress hormones on cognitive functions (Lupien et al., 1999). This research has revealed that working memory, a function thought to be very important for executive function (EF), is particularly sensitive to such effects (Lupien et al., 2007). Several studies show that stress exposure (Duncko et al., 2009; Weerda et al., 2010) or naturalistic stress (Lewis et al., 2008) can lead to improved performance on tests of working memory. However, other studies have demonstrated that the same types of stress exposure can cause 
impaired performance on working memory tasks (Oei et al., 2006; Schoofs et al., 2008, 2009; Luethi et al., 2009), as do higher levels of naturalistic stress (Sliwinski et al., 2006). Finally, a third set of research results show no differences in performance on working memory tasks between conditions of stress or no-stress exposure (Kuhlmann et al., 2005; Porcelli et al., 2008; Qin et al., 2009). What might explain these mixed findings?

One possibility is that these research endeavors have often focused on group differences (e.g., stress group versus no-stress group) without examining individual differences in response to those stressors (either subjective stress response or physiological reactivity). When researchers have monitored physiological reactivity to stress, several studies have found that cortisol and performance on working memory tasks are negatively correlated (Qin et al., 2009; Schoofs et al., 2009) especially when adrenergic activity is also high (Elzinga and Roelofs, 2005). Animal research has demonstrated that the effects of stress intensity on behavior are characterized by an inverted-U-shaped function: while low or high levels of stress lead to performance impairment on tests of vigilance and working memory, a moderate level of stress leads to performance improvement (Lupien et al., 2007; Arnsten, 2009). Neurobiological studies suggest that these quadratic effects are related to levels of stress hormones (e.g., glucocorticoids) and adrenergic activity (e.g., catecholamines) in the brain, especially in prefrontal cortex (PFC) regions (Arnsten and Goldman-Rakic, 1998; Mizoguchi et al., 2004; Lupien et al., 2007; Hains and Arnsten, 2008; Arnsten, 2009), which are critically involved in working memory and EF processes. Examining individual responses to stress, and how these responses relate to subsequent cognitive functioning (in either linear or quadratic relationships), is thus critical for clarifying the question of when stress may help or harm behavioral performance.

Controllability of stressors is also a key factor that influences how stress affects behavioral performance (Dickerson and Kemeny, 2004; Arnsten, 2009). Controllability is a characteristic of stress that has been explored in "learned helplessness" research. This research typically uses a triadic design in which two groups are exposed to equivalent stress but differ on whether or not it is possible to learn to control stressors, and a third (control) group is not required to learn control and usually is not exposed to stress. After these manipulations, participants are tested on learning or problem-solving tasks, and comparison of performance between groups reveals the effects of stress exposure and controllability. Learned helplessness research has provided evidence for the harmful effects of exposure to uncontrollable stress, as well as the protective effects of having behavioral control over stressors. Specifically, while exposure to uncontrollable stress leads to passivity, negative affect, and disrupted performance on subsequent cognitively demanding tasks, being able to learn how to behaviorally control the same stressor buffers the individual from these negative effects (see Maier and Seligman, 1976, for review). An extensive literature documents learned helplessness effects in a range of animal species (see Seligman, 1972; Maier, 1984 for reviews) and the neural mechanisms inhibiting the stress response under conditions of controllability are well defined in rodents (Maier and Watkins, 2005). Research with humans has replicated the behavioral effects of controllability, showing that uncontrollable situations, in which behavioral responses cannot affect outcomes, lead to poorer performance on subsequent learning and anagram tasks (Hiroto, 1974; Hiroto and Seligman, 1975; Jones et al., 1977; DeVellis et al., 1978; Hirt and Genshaft, 1981; Kofta and Sedek, 1989) and possibly a higher cortisol response (Peters et al., 1998).

Thus, controllability of, and individual responses to, stressors influence the effects of stress exposure on cognitive and behavioral functioning. However, several intriguing questions about the nature of these effects remain unexplored. First, the question of what types of stress exposure can enhance cognitive functioning remains only partially resolved. While some promising evidence suggests that exposure to moderately intense stress predicts improved working memory performance, the dearth of research on individual differences, and inconsistencies in grouplevel effects, make this association far from conclusive. Furthermore, the question remains open whether exposure to controllable stress can enhance behavioral functioning. A number of studies documented the impairing effects of uncontrollable stress, but failed to find any benefit of exposure to controllable stress (Hiroto, 1974; Hiroto and Seligman, 1975; Jones et al., 1977; DeVellis et al., 1978; Hirt and Genshaft, 1981). However, several other studies demonstrated that people exposed to controllable stress show improved learning and cognitive ability in comparison to people exposed to either uncontrollable stress or no-stress (Thornton and Jacobs, 1971; Thornton and Powell, 1974; Benson and Kennelly, 1976; Eisenberger et al., 1976, 1979). We have yet to determine whether, and how, controllable stress exposure may enhance cognitive and behavioral functioning.

Second, although controllability of stress and individual differences in stress response have been investigated separately, little research has examined the interaction of these factors. One research study showed that participants who appraised stressors as challenging performed better on an active coping task than participants who appraised the same stressors as threatening (Tomaka et al., 1993), suggesting that subjective reactions predict performance differences in a context of controllable stress. However, because the passive coping task used in this study for comparison had no measure of performance, it is not possible to determine whether subjective reaction would predict a different pattern in the context of uncontrollable stress. Another study identified an interaction between dosage of stress and individual differences in locus of control in predicting performance on an anagram task (Pittman and Pittman, 1979), although individual responses to stressors were not assessed. In sum, existing research supports the robust influences of controllability and stress reactivity in moderating the effect of stress on cognitively demanding tasks, but investigation of interactions between these factors remains sparse.

Third, our understanding of the cognitive abilities affected by stress controllability and level of stress response remains imprecise. Although both human and animal research suggests that the effects of controllability on cognitive function are mediated by underlying systems fundamental to learning (Maier and Watkins, 2005), the exact nature of those systems in humans is unclear. The most consistently replicated consequences of uncontrollable stress are impaired performance on novel, goal-directed learning tasks or complex tasks such as anagrams (reviews by Oakes, 1982; 
Tennen, 1982). Deficits on these complex tasks may have a number of sources ranging from slowed processing speed to reduced ability to direct and sustain attention. Hence, until research is conducted using tasks that isolate particular cognitive abilities, it is not possible to determine which abilities are affected by controllability and which are not. Several pieces of evidence suggest that EF may be the most likely underlying cognitive function impaired by exposure to uncontrollable stress. EF refers to a set of abilities including holding abstract goals in mind, using goals to provide "top-down" direction for attentional allocation, and inhibiting the processing of sensory information, thoughts or actions that are irrelevant to or incompatible with current goals. EFs are often recruited for tasks requiring complex cognition, including the various learning and problem-solving tasks that have been traditionally administered in stress controllability research. Of note, EF abilities are supported by systems in PFC, which is particularly sensitive to the effects of stress hormones and catecholamines (Arnsten, 2009).

Most of the research investigating effects of stress intensity or reactivity has focused on working memory ability. However, it is not known whether stress specifically affects working memory, or EF more broadly. EFs are best characterized as separable but related cognitive processes, with both unique and shared individual differences, genetic influences, and neural substrates (e.g., Miyake et al., 2000; Collette et al., 2005; Friedman et al., 2008). Impairment on working memory tasks could arise either from impairment of processes specific to working memory, or from impairment of processes common across multiple aspects of EF, namely the ability to actively maintain goal information (common $E F$; Friedman et al., 2008). Initial research supports the theory that stress affects executive abilities more broadly; researchers detected a larger difference between stressed and control subjects in performing a Sternberg working memory task when subjects had to ignore emotional distractors presented during a delay (Oei et al., 2009). In other research, scientists found that Trier stress exposure predicted impaired performance on tests of cognitive "flexibility" such as remote associates or anagrams tasks (Alexander et al., 2007) and that inducement or blockade of physiological arousal served to respectively impair or improve performance on such tasks (Beversdorf et al., 1999). Thus, stress appears to have effects across tasks that vary in the aspects of EF they tap, suggesting that stress may affect working memory abilities, at least in part, because stress affects processes that are common across EF tasks. This previous research suggests that common EF processes may be key cognitive functions affected by stress exposure.

To explore common EF processes in the current research, we administered the "gold standard" of EF tasks (MacLeod, 1992), the Stroop task (Stroop, 1935). In this task, participants must identify the color of ink in which a word is printed while ignoring the meaning of the word, which may contain competing color information (e.g., BLUE written in red ink). Hence, one must maintain the goal of identifying ink color in the face of highly distracting information (as in the competing color information conveyed by word meaning). Latent variable analysis has demonstrated that the Stroop task loads strongly on a common EF factor (Friedman et al., 2008), suggesting that this measure is suitable for examining general executive ability.
In sum, the current research investigated the helpful or harmful effects of stress on common EF, as assessed by the Stroop task. Specifically, this research explored the (linear or quadratic) relationship between individual differences in stress response and Stroop performance, and whether these effects depend on controllability of stress exposure.

\section{EXPERIMENT 1 \\ STUDY GOALS}

This study tested the hypothesis that controllability of stress, and individual differences in subjective stress, are factors that interact to affect EF. Our stress manipulation included two sources of psychological stress to which participants were exposed while performing a choice-RT task: social-evaluative stress in the form of performance pressure, and sensory stress in the form of noise exposure.

Previous research has shown that uncontrollable, socialevaluative stress is the most potent form of psychological stress (Dickerson and Kemeny, 2004). Researchers have defined uncontrollability as non-contingency between instrumental actions and outcomes (Oakes and Curtis, 1982), as repeated failure feedback regardless of responses (Klein et al., 1976; Jones et al., 1977; Kilpatrick-Tabak and Roth, 1978; Hirt and Genshaft, 1981) or as both non-contingency and failure (Hiroto, 1974; Hiroto and Seligman, 1975; Benson and Kennelly, 1976; Klein and Seligman, 1976; Miller and Seligman, 1976; Price et al., 1978; Kofta and Sedek, 1989). We structured the manipulation of uncontrollability to include both non-contingency and increased rates of failure. This decision was based on research indicating that this conjunction of stressors generates the most robust perception of uncontrollability, while the conjunction of true contingency and a high rate of success is the strongest generator of perceived control (Gernigon et al., 2000). This body of research also suggests that explicitly manipulating performance feedback makes the absence of contingency more obvious, and overrides the normal bias to assume one is controlling events when desired outcomes (here, shorter noises as opposed to longer noises) are frequent (Vallee-Tourangeau et al., 2005).

Social-evaluative stress occurs when an important aspect of self-identity is (or could potentially be) negatively judged by other people. Social-evaluative stress has been operationalized experimentally as pressure to succeed in active performance situations, e.g., on tasks that require overt or cognitive responses (Dickerson and Kemeny, 2004). We designed our stress manipulation to include social-evaluative stress by including trial-by-trial performance feedback, and by testing each participant individually in the presence of an experimenter.

As a result of the above considerations, we manipulated stress exposure between groups in the following manner. In our controllable stress condition (CSt), participants received accurate, feedback regarding their performance (fast responses elicited success feedback), and could learn to control the duration of noise stressors by responding quickly to stimuli. In our uncontrollable stress condition (USt), participants were exposed to two sources of uncontrollability. First, the type of noise and nature of performance feedback received for each trial were not contingent on responses. This non-contingency made it impossible for these 
participants to learn how to control the duration of the noise stress or reliably predict success versus failure feedback. Second, people in this group received a higher proportion of (inaccurate) failure feedback. This biased performance feedback suggested to participants that their responses were, overall, not fast enough to successfully perform the task. Importantly, the CSt and USt groups did not differ on noise exposure, task stimuli, or response requirements. Finally, we included a third, no-stress condition (NSt), in which people were required to respond to identical stimuli as the other two groups, but received no performance feedback or noise exposure.

In this study, participants completed the Stroop task at the beginning of the research session to assess their baseline ability to exert general EF. After completing one of the three stress task conditions described above, participants completed the Stroop task a second time. This design allowed us to control for baseline differences in EF by examining changes in Stroop performance from pre- to post-stress exposure.

\section{MATERIALS AND METHODS \\ Participants}

Participants were 109 undergraduate volunteers ages 18-24 from introductory psychology courses at the University of Colorado Boulder (Table 1). Participants provided informed consent and were treated in accordance with procedures approved by the University of Colorado Boulder Institutional Review Board. Participants were tested individually in private rooms.

\section{PROCEDURE}

Participants were informed of the study procedures at the beginning of the research session, provided written consent, and were randomly assigned to one of the three stress conditions (USt, $n=41$; CSt, $n=42$; NSt, $n=26)^{1}$. The sequence of an experimental sessions was: (1) PANAS-X pre-testing, (2) Stroop, (3) stress manipulation, (4) Stroop, (5) PANAS-X post-testing, (6) assessments of subjective stress response, perceived control, and Beck Depression Inventory II (BDI-II; Table 2). At the conclusion of the research session, all participants were given written debriefing

${ }^{1}$ Original recruitment included a sample size of 127 participants; however, 3 participants in the CSt condition and 1 participant in the USt condition failed to complete the experiment due to computer error, and 14 participants in the NSt condition failed to complete self-report measures due to experimenter error. For these reasons, analyses were performed on a sample size of $n=109$. information. Participants exposed to uncontrollable stress were debriefed verbally about such uncontrollability.

\section{Materials}

A computer system captured accuracy and reaction time (RT) via millisecond-accurate keyboard press for all trials. Participants assigned to stress conditions that included an auditory stressor (either 2000 or $4000 \mathrm{~ms}$ in duration) listened to stimuli through headphones, with volume calibrated at $72-80 \mathrm{~dB}$.

Test of executive functioning: color-word Stroop. On each trial of the Stroop task a word written in one of four ink colors (green, yellow, red, or blue) appeared in the center of the screen for $2000 \mathrm{~ms}$ and participants identified the ink color as quickly as possible by hitting the corresponding button on the keyboard. Prior to beginning the first Stroop task, participants were given 16 practice trials in which XXXX stimuli were presented to familiarize the participant with the task demands and location of the response keys for each of the four colors. During the task, trials were presented in two blocks ( 48 trials each; $38 \%$ incongruent and $62 \%$ neutral across blocks). Incongruent words feature conflict between ink color and word meaning (e.g., RED written in blue ink), while neutral (non-color) words do not (e.g., SUM written in blue ink). Comparing RT to incongruent versus neutral words isolates the individual's ability to exert cognitive control in the face of highly distracting information, over and above basic perceptual processing abilities and response speed. Therefore, the calculation of percent difference in incongruent versus neutral RT [(incongruent RT - neutral RT)/neutral RT] yields an interference score that indexes general executive functioning. This method of calculating Stroop interference (as a percentage of neutral trial RT) controls for scaling effects in RT measures, in which RT differences tend to scale with the magnitude of RT latency (Lansbergen et al., 2007).

Stress manipulation. Participants performed a choice-RT task and either were (USt and CSt groups) or were not (NSt group) exposed to concurrent psychological stress. The choice-RT task required participants to choose behavioral responses based on perceptual features in the display (Figure 1).

For each trial, an arrow pointing either left or right appeared inside a white fixation box on the computer monitor. Participants responded to the direction of the arrow as quickly as possible by pressing the corresponding button on the keyboard. All

Table 1 | Demographics and descriptive statistics for Experiment 1.

\begin{tabular}{|c|c|c|c|c|c|c|c|}
\hline \multirow[t]{2}{*}{ Condition } & \multirow{2}{*}{$\begin{array}{l}\text { Sample } \\
n \text { ( } n \text { female) }\end{array}$} & \multicolumn{3}{|c|}{ Self-report } & \multicolumn{3}{|c|}{ Cognitive tasks } \\
\hline & & $\begin{array}{l}\text { Subjective } \\
\text { stress } \\
M \text { (SD) }\end{array}$ & $\begin{array}{l}\text { Subjective } \\
\text { control } \\
M \text { (SD) }\end{array}$ & $\begin{array}{l}\text { BDI score } \\
M \text { (SD) }\end{array}$ & $\begin{array}{l}\text { Pre-stress Stroop } \\
\text { interference } \\
M \text { (SD) }\end{array}$ & $\begin{array}{l}\text { Post-stress Stroop } \\
\text { interference } \\
M \text { (SD) }\end{array}$ & $\begin{array}{l}\text { Change in } \\
\text { interference } \\
M \text { (SD) }\end{array}$ \\
\hline CSt & $42(29)$ & $17.07(4.67)$ & $6.71(1.47)$ & $11.51(6.54)$ & $0.0119(0.0101)$ & $0.0074(0.0094)$ & $-0.0045(0.0108)$ \\
\hline Total & 109 (63) & $17.16(5.00)$ & $5.53(2.04)$ & $10.15(7.11)$ & $0.0118(0.0103)$ & $0.0090(0.0092)$ & $-0.0027(0.0106)$ \\
\hline
\end{tabular}


participants completed a practice block (20 trials) without any stress exposure, to familiarize themselves with the choice-RT task.

In the two testing blocks ( 80 trials each), participants in the CSt and USt groups had two performance goals: (1) to respond accurately and fast enough to beat a challenging time limit, for which they received performance feedback indicating success (yellow fixation box) or failure (blue fixation box; blocks 1 and 2); and (2) to

Table 2 | Experimental procedure (Experiments 1 and 2).

Task or measure

Time (minute)

\section{EXPERIMENT PROCEDURE}

Rating of state affect (PANAS-X)

Assess baseline general executive functioning

5

(color-word Stroop)

Stress manipulation

16

Assess post-stress general executive functioning

5

(color-word Stroop)

Rating of state affect (PANAS-X)

2

Rating of subjective stress, control

2

Report depression (BDI-II)

5

Total learn how their responses controlled the duration of a noise stressor that was evoked by each response (block 2 only). Participants in the NSt condition completed the same task, but with no feedback or noise stress. This task was based on classic manipulations of instrumental control in which participants must learn how to control a noise by pushing a sequence of buttons (Hiroto, 1974; Hiroto and Seligman, 1975).

For the CSt group, feedback and noise exposures were controllable: fast, accurate responses elicited short noises accompanied by success feedback, while slow or inaccurate responses elicited long noises coupled with failure feedback. A moving-window for response speed ensured that every participant was able to beat the time limits on $80 \%$ of trials, and participants received success feedback and short noises on these trials. When participants failed to beat the time limit ( $20 \%$ of trials) they received failure feedback and long noises.

For the USt group, feedback and noise exposures were uncontrollable, both (1) because feedback and noises were not contingent on response speed, and (2) because feedback was biased to indicate a higher rate of failure (blue fixation box for $50 \%$ of trials, regardless of response speed or accuracy). The CSt and USt groups were matched on their true response success and noise exposure: as in the CSt group, a moving-window for response speed ensured that every participant was actually able to beat the time limits

\begin{tabular}{|c|c|c|c|}
\hline \multicolumn{4}{|c|}{ STRESS MANIPULATION } \\
\hline & \multicolumn{3}{|c|}{ GROUP } \\
\hline BLOCK & $\begin{array}{l}\text { No Stress } \\
\text { (NSt) }\end{array}$ & Controllable Stress (CSt) & Uncontrollable Stress (Ust) \\
\hline Practice & & & $>$ \\
\hline feedback & none & none & none \\
\hline Block 1 & & - & \\
\hline feedback & none & $\begin{array}{cc}\text { failure } & \text { success } \\
\text { (slow response) } & \text { (fast response) }\end{array}$ & $\begin{array}{c}\text { failure success } \\
\text { (type of feedback unrelated to } \\
\text { response speed) }\end{array}$ \\
\hline Block 2 & & & $>$ \\
\hline feedback & none & $\begin{array}{cc}\text { failure } & \text { success } \\
\text { (slow response) } & \text { (fast response) }\end{array}$ & $\begin{array}{l}\text { failure success } \\
\text { (type of feedback unrelated to } \\
\text { response speed) }\end{array}$ \\
\hline $\begin{array}{l}\text { noise } \\
\text { stress }\end{array}$ & none & $\begin{array}{cc}\text { LONG } & \text { SHORT } \\
\text { (slow response) } & \text { (fast response) }\end{array}$ & $\begin{array}{l}\text { (type of noise unrelated to } \\
\text { response speed OR type of } \\
\text { feedback) }\end{array}$ \\
\hline
\end{tabular}

FIGURE 1 | Stress manipulation: In both Experiments 1 and 2, all groups completed a simple choice-RT task that either was accompanied by psychological stress in the form of performance feedback and noise exposure (controllable stress: CSt group and uncontrollable stress: USt group) or was not accompanied by these forms of stress (no-stress: NSt group). The manipulation consisted of a practice block that was identical across groups, followed by two testing blocks that varied between groups. Performance feedback (blocks 1 and 2): the NSt group received no performance feedback; the CSt group received accurate feedback indicating success or failure in responding fast enough to beat a time limit; the USt group received performance feedback that was unrelated to their response speed and either featured an exaggerated proportion of failure feedback (Experiment 1) or was equated on feedback with the CSt group (Experiment 2). Noise exposure (block 2): the NSt group received no noise exposure; the CSt group was able to learn that short noises were contingent on responding fast enough to beat time limits; the USt group was exposed to non-contingent noises unrelated to response speed or performance feedback, and the amount of short and long noises were equated with the CSt group. 
on $80 \%$ of trials, and every participant received a short noise on $80 \%$ of trials. However, unlike the CSt group, the USt participants received non-contingent performance feedback that was biased for failure (success feedback for only 50\% of trials) and short and long noises were random and unrelated to their response speed or performance feedback ${ }^{2}$.

Finally, for the NSt group, participants received no performance feedback (green fixation box after every response, regardless of response accuracy or speed) and were not exposed to noise (Figure 1).

Assessment of subjective stress response. At the end of testing, participants reported subjective ratings of stress to provide a measure of individual differences in response to the stress exposure. Participants rated the following on a 1 (low) to 9 (high) scale: (1) level of stressfulness of the noise exposures (CSt and USt only), (2) level of stressfulness of the task (choice-RT) demands, (3) degree to which you believe someone else would have performed better than you (social comparison), (4) degree to which you believe you performed well on the task. The scores for these scales were summed to yield a composite score of subjective stress for each participant. Because they did not rate the noise exposure item, scores for participants in the NSt condition were multiplied by $4 / 3$ to make this group comparable to the CSt and USt groups. Across the sample, scores were mean-deviated for the purpose of regression analyses.

Assessment of perceived control. Self-reported perception of control was also assessed at the end of testing, with ratings reported from 1 (low) to 9 (high). This measure was collected to confirm that our manipulation of controllability was successful in eliciting differences between groups.

Assessment of mood and affect. We used well-validated measures of current depression and state affect to confirm that groups were equivalent on these dimensions at baseline, and to investigate whether participants experienced changes in affect over the course of testing.

Participants reported level of depression in the past two weeks using the BDI-II (Beck et al., 1996). Previous research has shown that current depression can moderate the effects of controllable stress on cognitive task performance (Klein et al., 1976; Miller and Seligman, 1976; Price et al., 1978). In addition, sex differences in physiological and psychological responses to stress are consistently noted in stress research (e.g., Ordaz and Luna, 2012). Therefore, for all analyses, we also conducted regressions including BDI-II score and participant sex as covariates. Unless otherwise indicated, the significance of the results was not

\footnotetext{
${ }^{2}$ We expected that response speed on choice-RT trials would vary between groups, given that the no-stress group did not receive performance feedback and therefore may have been less motivated to respond quickly on choice-RT trials. Analyses revealed an effect of stress exposure, in which the NSt group had slower response times in both testing blocks than the CSt or USt group ( $p$ 's $<0.001)$, who did not differ from one another $(p$ 's $>0.2)$. However, response speed on choice-RT trials was unrelated to subjective stress ( $p$ 's $>0.1$ ) or Stroop interference ( $p$ 's $>0.5$ ), and including choice-RT speed as a covariate in the Stroop analyses failed to alter any statistical effects. Therefore we report simple analyses only.
}

altered by the addition of these covariates and we report simple analyses only.

In addition, they completed the Positive and Negative Affect Questionnaire (PANAS-X; Watson and Clark, 1999) both before and after cognitive testing as a measure of state affect.

\section{RESULTS}

\section{Data processing and analyses}

For the Stroop tasks, RT analyses were conducted by calculating an average for each trial type. Incorrect trials and trials on which RTs were less than $200 \mathrm{~ms}$ or exceeded 3 standard deviations above the within-subject mean were excluded from analyses. RTs were natural log transformed to reduce the skew common to RT data and which violates the statistical assumption of normal distribution necessary for analysis. Accuracy analyses were conducted by calculating the total correct for each trial type pre- and post-stress manipulation.

Data were analyzed with multiple-regression analyses. For group comparisons, two orthogonal contrast-coded predictors were entered in the regression model: controllability $(\mathrm{CSt}=1$, $\mathrm{USt}=-1, \mathrm{NSt}=0)$ and stress exposure $(\mathrm{CSt}=-1, \mathrm{USt}=-1$, $\mathrm{NSt}=2$ ). For group by subjective stress response (linear or quadratic) interactions, these contrast codes were multiplied by the subjective stress score (controllability $\times$ subjective stress and stress exposure $\times$ subjective stress) or square [controllability $\times(\text { subjective stress })^{2}$ and stress exposure $\times$ (subjective stress $\left.)^{2}\right]$.

Outlier detection was accomplished in two ways: (1) observations on self-report measures that exceeded 3 standard deviations above or below the group mean were excluded from analyses; (2) for any significant regression effects, standardized df beta was calculated to detect observations that had undue influence on the analysis according to the standard threshold (df beta $>2 /(\sqrt{ } n)$ ).

\section{Effects of controllability on perceived control and subjective stress}

As a manipulation check, we compared self-reported perceived control between the CSt and USt groups (note: members of the NSt group did not rate this item, as they were not asked to learn to control outcomes). Confirming that the controllability manipulation was effective, the CSt group $(M=6.71)$ reported a higher level of perceived control during the stress manipulation than the USt group $(M=4.32), t(1,81)=6.57, p<0.001, R^{2}=0.35$.

We conducted analyses to examine whether our stress manipulation affected subjective ratings of stress. Including sex as a covariate revealed a significant difference in subjective stress responses between men and women, across groups; women reported higher stress $(M=19.44)$ than men $(M=13.43), F(1,98)=35.09$, $p<0.001, R^{2}=0.26$. Controlling for sex, there was a significant effect of stress exposure on subjective ratings of stress. Participants who were not exposed to noise or performance pressure stress (i.e., the NSt group) reported lower subjective stress $(M=14.56)$ than participants exposed to stress $(M=18.05), F(1,98)=4.79$, $p=0.031, R^{2}=0.05$. However, there was a no effect of controllability on subjective stress, $F(1,98)=1.62, p=0.21$, and no interactions between sex and stress exposure or stress controllability ( $p$ 's $>0.34$ ). These analyses suggest that female participants experienced our research task as being more stressful than male 
participants, but that the nature of the relationships between controllability and subjective stress were similar between the sexes.

Finally, because subjects in the uncontrollable stress group received a higher rate of failure feedback, we might expect their performance ratings to be more negative than the ratings of subjects in the other groups. This is reflected in their slightly higher composite ratings of subjective stress (see Table 1), although as noted above, when controlling for sex differences in stress response, the difference in subjective stress between controllability groups was not significant. Furthermore, because our analyses were conducted via multiple-regression, in which the effect of each predictor variable is detected over and above variance shared between predictors, such relationships between stress condition and subjective response variables are controlled. However, we also conducted analyses using a revised subjective stress composite that only included ratings of noise and task stress. The pattern of results was consistent with that reported above: there was a significant difference between men and women's reports of subjective stress $(p<0.001)$, and a significant effect of stress exposure on subjective stress $(p=0.01)$ but no effects of controllability or interactions with sex ( $p$ 's $>0.37)$. Moreover, analyses of stress effects on executive functioning using the revised subjective stress composite measure yielded the same pattern of results as those with the full 4-scale composite measure. Because these patterns remained consistent, and due to the higher reliability of a composite stress measure that includes four, as compared with two, rating scales, all subsequent analyses used the full 4-scale composite measure.

\section{Effects of stress controllability and subjective stress on executive functioning}

We conducted a regression predicting changes in Stroop interference by the following: group contrast codes (controllability and stress exposure), the linear effect of subjective stress, the quadratic effect of subjective stress, and interactions between group predictors and stress response effects (Table 3 ). All effects are controlling for all other variables in the regression model.
There was a significant difference in Stroop interference changes between the controllable and uncontrollable stress groups, $F(1,98)=7.76, p=0.006, R^{2}=0.07$. This result indicates that when participants are equated on subjective stress, exposure to controllable stress is related to greater improvements in Stroop performance than exposure to uncontrollable stress. In addition, there was a significant quadratic relationship between subjective stress and changes in Stroop interference, $F(1,98)=5.33, p=0.023, R^{2}=0.05$. While low and high levels of subjective stress were related to increased interference, a moderate level of subjective stress was related to reduced interference (improved Stroop performance).

There was a significant interaction between stress controllability and the quadratic effects of subjective stress in predicting change in Stroop interference, $F(1,98)=5.37, p=0.023$, $R^{2}=0.05$. This result indicates that the quadratic relationship between subjective stress and Stroop performance varies between the controllable and uncontrollable stress conditions. Follow-up analyses were conducted to determine the nature of this difference. Specifically, there was a significant quadratic relationship within the CSt group, $F(1,38)=7.72, p=0.008$, $R^{2}=0.17$, showing that while low or high levels of subjective stress were related to impaired Stroop performance, moderate levels of subjective stress were related to improved Stroop performance. In contrast, there was no quadratic relationships between subjective stress and interference change within the USt group, $F(1,38)=0.064, p=0.8$, or within the NSt group, $F(1,23)=0.088$, $p=0.7$ (Figure 2).

We conducted analyses to confirm that baseline differences in Stroop performance did not drive the effects of group noted above. A regression predicting baseline interference scores by group contrast codes confirmed that there were no significant differences in Stroop performance between subjects randomly assigned to each of the three stress conditions, $F(2,106)=0.48, p=0.6$.

We conducted analyses to investigate whether experimental groups differed in Stroop accuracy, either at baseline or over the

Table 3 | Regression table for Experiment 1, model predicting change in Stroop interference (post-pre stress manipulation) by group status (controllability: compares CSt versus USt groups; stress exposure: compares NSt group versus the average across CSt and USt groups); subjective stress response (subjective stress: the linear effect of subjective stress; subjective stress ${ }^{2}$ : the quadratic effect of subjective stress); and interactions between these factors.

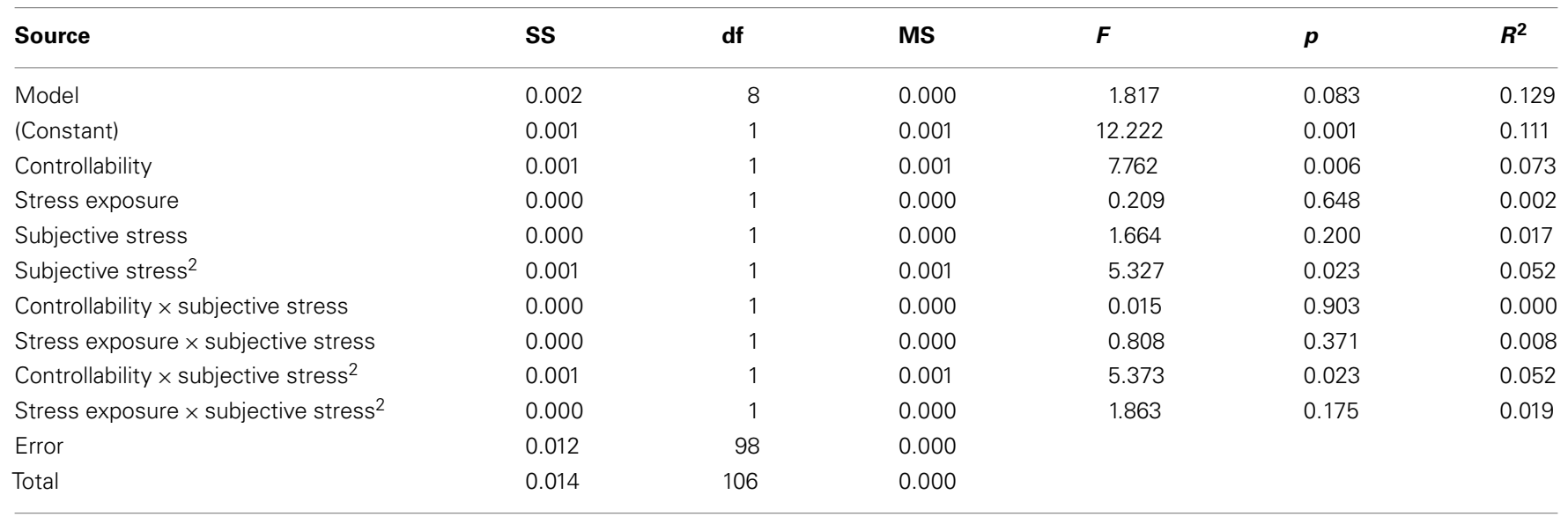

${ }^{a} R^{2}=0.129$ (adjusted $R^{2}=0.058$. 
A Stroop interference across groups

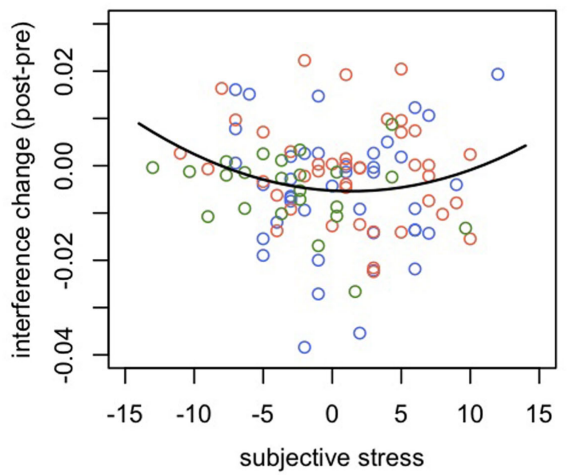

C Stroop interference USt group

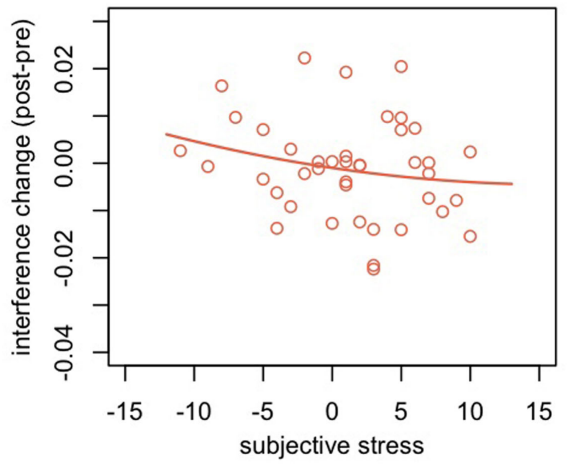

FIGURE 2 | Experiment 1: Change in Stroop interference predicted by subjective stress and controllability. Change in Stroop interference

(post-pre stress manipulation) predicted by individual differences in subjective stress and by group. (A) Quadratic relationship between subjective stress and change in Stroop interference across all groups. (B) Quadratic relationship

\section{B Stroop interference CSt group}

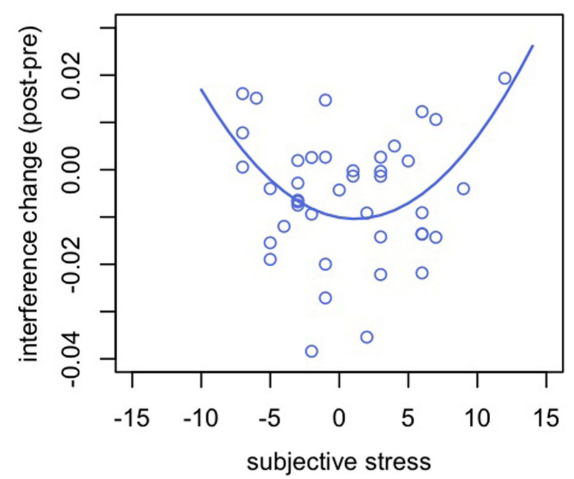

D Stroop interference NSt group

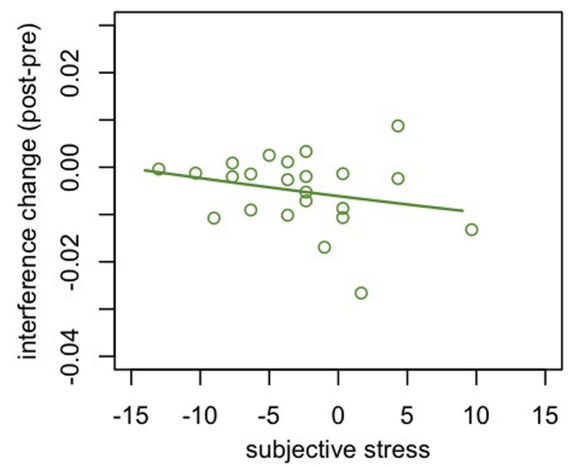

between subjective stress and change in Stroop interference within the group of participants with behavioral control over stressors. (C) Absence of a significant relationship between subjective stress and Stroop interference changes for the group of people exposed to uncontrollable stress, or (D) to no-stress. course of testing. There were no differences in baseline incongruent or neutral trial accuracy between groups ( $p$ 's $>0.15$ ). Next we examined changes in incongruent trial accuracy from before to after the stress manipulation. There was no effect of stress exposure on changes in incongruent trial accuracy: subjects in the no-stress group showed no changes on this measure $(M=-0.19)$, and their performance did not differ from that of subjects exposed to stress $(M=-0.32), F(1,106)=0.24, p=0.8$. However, there was a significant difference detected between controllability groups: subjects who were exposed to controllable stress showed more of an improvement in accuracy on incongruent trials $(M=0.26)$ than subjects exposed to uncontrollable stress, who showed a slight decrease in accuracy $(M=-0.88)$, $F(1,106)=4.71, p=0.03, R^{2}=0.04$. There were no changes in neutral trial accuracy over the course of testing, and no differences in such accuracy change between groups ( $p$ 's $>0.7$ ).

We conducted a full regression in which changes in incongruent trial accuracy were predicted by group contrast codes, the linear and quadratic effects of subjective stress, and interactions between these variables. This analysis revealed a marginal difference in accuracy change between the CSt and USt groups in which having behavioral control predicted a greater improvement in incongruent trial accuracy, $F(1,99)=2.80, p=0.09, R^{2}=0.03$.

\section{Mood and affect}

All groups showed similar positive affect $(M=28.63)$, $F(2,106)=0.004, \quad p=0.9$, and negative affect $(M=14.68)$, $F(2,106)=1.57, p=0.2$, at baseline. We also conducted analyses to investigate whether changes in affect (post-testing affect - pretesting affect) over the course of testing were significantly different between groups. On average, participants reported minimal changes in negative $(M=-0.66)$ and positive affect $(M=-5.88)$, and there were no differences between groups, $F(2,106)=1.16$, $p=0.3$ and $F(2,106)=1.37, p=0.3$. In addition, all groups showed comparable, and low, current levels of depression as assessed by the BDI-II, $(M=10.15), F(2,105)=1.41, p=0.2$.

\section{DISCUSSION}

The results of Experiment 1 suggest that controllability of stress, as well as individual differences in subjective response to stress, together influence the impact of stress exposure on executive functioning in the color-word Stroop. Specifically, these results show that characteristics of stress exposure such as controllability and subjective response can cause stress to have either beneficial or harmful effects on cognitive abilities. Exposure to controllable stress improved executive functioning only when that stress was experienced as moderate. In contrast, stress response had 
no relationship with executive functioning when stress exposure was uncontrollable. Furthermore, when equating participants on levels of subjective stress, those who learned to control stressors showed improved Stroop performance compared to those exposed to uncontrollable stress.

Although these results suggest the importance of controllability in moderating the effects of stress on EF, it is not clear from this study which aspects of controllability are essential to this relationship. The question of specifying which aspects of controllability actively drive effects has been controversial. Some studies have suggested that differing rates of failure account completely for learned helplessness effects (Matute, 1994, 1995), while others have demonstrated that non-contingency is the crucial factor, and failure is unnecessary, in evoking learned helplessness (Oakes and Curtis, 1982; Tennen et al., 1982b; Kofta and Sedek, 1989). A third body of research suggests that these factors have additive or interactive effects on behavior (Koller and Kaplan, 1978; Tennen et al., 1982a). In considering the results of Experiment 1, one possibility is that the ability to learn contingencies between actions and outcomes caused moderately reactive participants to respond adaptively to controllable stress, while the inability to learn contingencies eradicated this relationship for people exposed to uncontrollable stress. Another possibility is that differences in performance feedback contributed to the moderating effect of controllability: people exposed to uncontrollable stress received biased feedback indicating a higher rate of failure, which may have caused them to feel discouraged or lose motivation (although all groups reported similar affect before and after testing). With the goal of clarifying which aspects of uncontrollability generated the effects observed in Experiment 1, we conducted Experiment 2, in which rates of failure feedback were held constant across groups exposed to stress.

\section{EXPERIMENT 2 \\ STUDY GOALS}

To test the hypothesis that non-contingency between behavioral actions and stressors is critical to the effects we observed in Experiment 1 , we equated our controllable and uncontrollable stress groups on performance feedback, so that participants in both groups would receive the same total amount of success and failure feedback over the course of testing. However, it was once again impossible for participants in the uncontrollable condition to learn how to control stressors or accurately anticipate the type of feedback they received for each trial.

\section{METHOD}

\section{Participants}

Participants were 90 undergraduate volunteers ages 18-24 from introductory psychology courses at the University of Colorado Boulder (Table 4). Participants provided informed consent and were treated in accordance with procedures approved by the University of Colorado Boulder Institutional Review Board. Participants were randomly assigned to one of three stress conditions (USt, $n=28$; CSt, $n=30$; NSt, $n=32)^{3}$.

\section{Procedure}

Study procedures were the same as in Experiment 1.

\section{Materials}

Individual testing and materials were the same as in Experiment 1 , except as noted.

Stress manipulation. The stress manipulation was modified for Experiment 2 to hold the rates of success and failure feedback constant across CSt and USt groups. Every research participant responded to identical choice-RT trials, and a moving-window for response speed ensured that everyone was able to beat the time limits on $70 \%$ of trials. Again, the NSt group never received performance feedback (green fixation box after every response). The CSt participants received accurate, response-contingent performance feedback (success feedback for $70 \%$ of trials), and received short noises on successful trials (70\%) and long noises on failed trials (30\%). The USt participants received an identical rate of success feedback $(70 \%)$ that was, however, not contingent on response speed, and were exposed to short (70\%) and long (30\%) noises that were unrelated to their response speed or to performance feedback. Noise exposures were once again equated between the CSt and USt groups, so participants in these groups received the same total number of short and long noises.

Participants in the CSt and USt groups completed a practice block (20 trials) without noise exposure or feedback, a feedback block ( 60 trials) that included performance feedback as described above but no noise exposure, and a stress block (60 trials) that included performance feedback and noise exposure as described

\footnotetext{
${ }^{3}$ Recruitment included an additional two participants in the USt group, however, these participants failed to complete the experiment due to (1) computer error, and (2) one participant reported color-blindness that caused him to be unable to perform the Stroop task.
}

Table 4 | Demographics and descriptive statistics for Experiment 2.

\begin{tabular}{|c|c|c|c|c|c|c|c|}
\hline \multirow[t]{2}{*}{ Condition } & \multirow{2}{*}{$\begin{array}{l}\text { Sample } \\
n \text { ( } n \text { female) }\end{array}$} & \multicolumn{3}{|c|}{ Self-report } & \multicolumn{3}{|c|}{ Cognitive tasks } \\
\hline & & $\begin{array}{l}\text { Subjective } \\
\text { stress }\end{array}$ & $\begin{array}{l}\text { Subjective } \\
\text { control } \\
M \text { (SD) }\end{array}$ & $\begin{array}{l}\text { BDI score } \\
M \text { (SD) }\end{array}$ & $\begin{array}{l}\text { Pre-stress Stroop } \\
\text { interference } \\
M \text { (SD) }\end{array}$ & $\begin{array}{l}\text { Post-stress Stroop } \\
\text { interference } \\
M \text { (SD) }\end{array}$ & $\begin{array}{l}\text { Change in } \\
\text { interference } \\
M \text { (SD) }\end{array}$ \\
\hline CSt & $30(18)$ & $17.13(4.32)$ & $5.57(1.33)$ & $9.20(6.78)$ & $0.0159(0.0091)$ & $0.0105(0.0090)$ & $-0.0054(0.0101)$ \\
\hline Total & $90(48)$ & $17.16(5.00)$ & $4.14(2.54)$ & $9.97(6.17)$ & $0.0149(0.0120)$ & $0.0094(0.0098)$ & $-0.0055(0.0135)$ \\
\hline
\end{tabular}


above. These trial blocks were shorter in Experiment 2 to minimize the amount of noise exposure per participant, and equate the amount of long noises (reported in pilot testing as considerably more stressful) experienced by each participant across Experiments 1 and 2. Participants in the NSt group completed the same task but did not receive feedback or noise stress during any block ${ }^{4}$.

Other measures. All assessments of executive functioning, mood, affect, and subjective stress response, were conducted in Experiment 2 with the same measures as implemented in Experiment 1. Again, we also conducted regressions including BDI-II score and participant sex as covariates. Unless otherwise indicated, the significance of the results was not altered by the addition of these covariates and we report simple analyses only.

\section{RESULTS}

\section{Data processing and analyses}

Data processing and analyses were the same as for Experiment 1.

\section{Effects of controllability on perceived control and subjective stress}

Confirming that the controllability manipulation was effective, the CSt group $(M=5.57)$ reported a higher level of perceived control during the stress task than the USt group $(M=2.61)$, $t(1,56)=5.44, p<0.001, R^{2}=0.35$.

Next we investigated the effects of stress exposure and controllability on subjective stress responses. Again, including sex as a covariate revealed a significant difference in subjective stress responses between men and women, across groups; women

\footnotetext{
${ }^{4}$ As in Experiment 1, the NSt group had slower response times in both testing blocks than the CSt or USt group ( $p$ 's $<0.001$ ), as expected. In the second testing block, the CSt group had faster response times than the USt group $(p=0.03)$ but this difference did not emerge in the first testing block $(p=0.8)$. Response speed on choice-RT trials was unrelated to subjective stress ( $p$ 's $>0.1$ ) or Stroop interference ( $p$ 's $>0.3$ ), and including choice-RT speed as a covariate in the Stroop analyses failed to alter any statistical effects. Therefore we report simple analyses only.
}

reported higher stress $(M=17.20)$ than men $(M=13.46)$, $F(1,79)=12.04, p=0.001, R^{2}=0.13$. Controlling for sex, participants who were not exposed to noise or performance pressure stress reported lower levels of stress $(M=12.99)$ than participants who were exposed to stress (CSt, $M=17.13$ and USt, $M=15.26$ ), $F(1,85)=4.44, p=0.038, R^{2}=0.05$. However, there was no effect of controllability on subjective stress, and no interactions between sex and stress exposure or controllability ( $p$ 's $>0.14)$.

\section{Effects of stress controllability and subjective stress on executive functioning}

To examine the interactive effects of stress controllability and subjective stress on executive functioning, we conducted regression analysis in which change in Stroop interference was predicted by group contrast-coded predictors, subjective stress (both linear and quadratic effects), and the interactions of these predictors (Table 5). All effects are controlling for all other variables in the regression model.

There was a significant interaction between stress controllability and the linear effect of subjective stress, $F(1,17)=5.00$, $p=0.028, R^{2}=0.061$. This result indicates, again, that the relationships between subjective stress and Stroop performance varied by controllability of stress exposure. However, in this analysis, it was the linear effect of stress that varied between groups. Followup analyses were conducted to determine the nature of the linear effects of subjective stress within groups. Within the CSt group, there was a significant linear effect of stress on Stroop interference such that at higher levels of subjective stress, performance became impaired, but at moderate levels of stress, performance was improved $F(1,27)=6.92, p=0.014, R^{2}=0.20$. There were no linear or quadratic relationships between subjective stress and interference change within the USt group, $F(1,23)=0.89, p=0.4$, or within the NSt group, $F(1,27)=0.88, p=0.4$ (Figure 3 ).

In the full regression described above, there were no quadratic effects of subjective stress detected when controlling for group

Table 5 | Regression table for Experiment 2, model predicting change in Stroop interference (post-pre stress manipulation) by group status (controllability: compares CSt versus USt groups; stress exposure: compares NSt group versus the average across CSt and USt groups); subjective stress response (subjective stress: the linear effect of subjective stress; subjective stress ${ }^{2}$ : the quadratic effect of subjective stress); and interactions between these factors.

\begin{tabular}{|c|c|c|c|c|c|c|}
\hline Source & SS & df & MS & $\boldsymbol{F}$ & $\boldsymbol{P}$ & $R^{2}$ \\
\hline Model & $0.002^{a}$ & 8 & 0.000 & 1.092 & 0.378 & 0.102 \\
\hline (Constant) & 0.003 & 1 & 0.003 & 16.933 & 0.000 & 0.180 \\
\hline Controllability & 0.000 & 1 & 0.000 & 0.229 & 0.633 & 0.003 \\
\hline Stress exposure & 0.000 & 1 & 0.000 & 0.160 & 0.691 & 0.002 \\
\hline Subjective stress & 0.000 & 1 & 0.000 & 0.052 & 0.820 & 0.001 \\
\hline Subjective stress ${ }^{2}$ & 0.000 & 1 & 0.000 & 0.359 & 0.551 & 0.005 \\
\hline Controllability $\times$ subjective stress & 0.001 & 1 & 0.001 & 4.991 & 0.028 & 0.061 \\
\hline Stress exposure $\times$ subjective stress & 0.000 & 1 & 0.000 & 0.371 & 0.544 & 0.005 \\
\hline Controllability $\times$ subjective stress ${ }^{2}$ & 0.000 & 1 & 0.000 & 0.035 & 0.852 & 0.000 \\
\hline Stress exposure $\times$ subjective stress ${ }^{2}$ & 0.000 & 1 & 0.000 & 0.011 & 0.918 & 0.000 \\
\hline Error & 0.014 & 77 & 0.000 & & & \\
\hline Total & 0.016 & 85 & 0.000 & & & \\
\hline
\end{tabular}

${ }^{a} R^{2}=0.102$ (adjusted $R^{2}=0.009$ ). 
A Stroop interference across groups

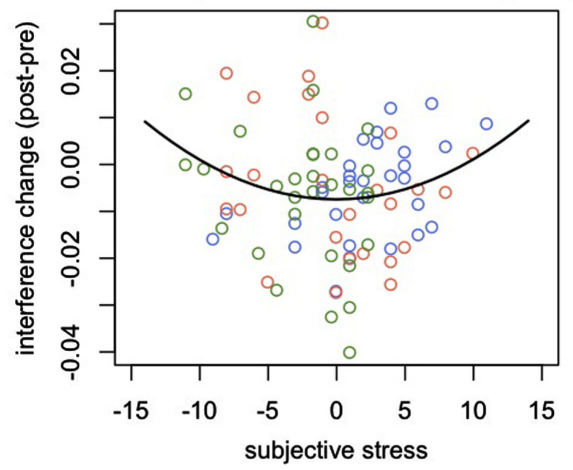

C Stroop interference USt group

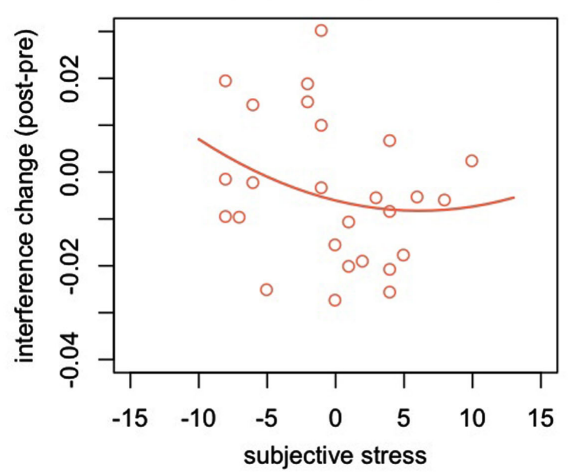

FIGURE 3 | Experiment 2: Change in Stroop interference predicted by stress reactivity and controllability. Change in Stroop interference (post-pre stress manipulation) predicted by individual differences in subjective stress and by group. (A) Quadratic relationship between subjective stress and change in Stroop interference across all groups. (B) Linear relationship

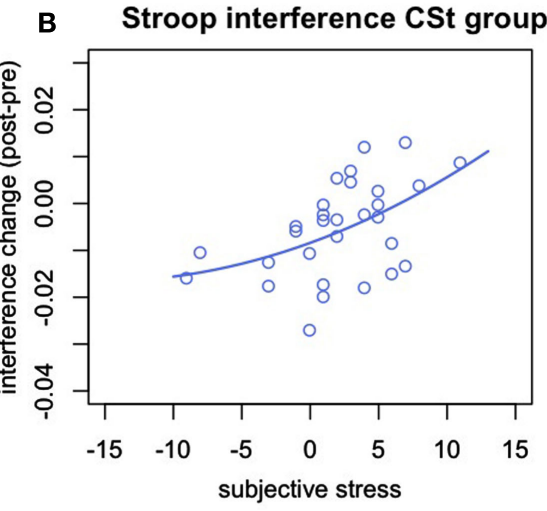

D Stroop interference NSt group

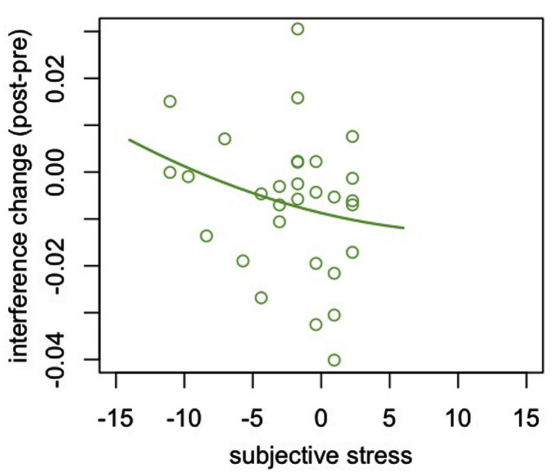

between subjective stress and change in Stroop interference within the group of participants with behavioral control over stressors. (C) Absence of a significant relationship between subjective stress and Stroop interference changes for the group of people exposed to uncontrollable stress, or (D) to no-stress. and group interactions. This result could indicate that different groups clustered on different parts of the quadratic curve, so that equating participants on group status simply washed out the quadratic effect. In consideration of this possibility, and to examine the effects of stress response on executive functioning across groups, we conducted a regression predicting change in Stroop interference by the linear and quadratic effects of subjective stress. Examined this way, there was a significant quadratic effect such that moderate levels of stress predicted reduced Stroop interference while low or high levels of subjective stress predicted increased Stroop interference, $F(1,86)=4.27, p=0.042, R^{2}=0.047$.

To determine whether there were group differences in Stroop performance at baseline that could contribute to these effects, we regressed baseline interference scores on group contrast codes. This analysis confirmed that participants randomly assigned to each of the three stress conditions showed comparable Stroop interference at baseline, $F(2,87)=1.10, p=0.4$.

We conducted analyses to investigate whether experimental groups differed in Stroop accuracy. There were no differences in baseline incongruent or neutral trial accuracy between groups ( $p$ 's $>0.2$ ). In addition, there was no effect of stress exposure on incongruent trial accuracy: the accuracy of subjects in the no-stress group did not change over testing $(M=0.03)$, and their performance did not differ from subjects exposed to stress $(M=0.55), F(1,86)=0.53, p=0.5$. However, there was a significant difference between controllability groups: subjects who were exposed to controllable stress showed an improvement in accuracy on incongruent trials $(M=1.13)$ while subject exposed to uncontrollable stress showed no change in accuracy $(M=-0.04)$, $F(1,86)=4.33, p=0.04, R^{2}=0.05$. There were no changes in neutral trial accuracy over the course of testing, and no differences in neutral trial accuracy change between groups ( $p$ 's $>0.6$ ).

We conducted a full regression in which changes in incongruent trial accuracy were predicted by group contrast codes, the linear and quadratic effects of subjective stress, and interactions between these variables. This analysis revealed a significant difference in accuracy change between the CSt and USt groups in which having behavioral control predicted a greater improvement in incongruent trial accuracy, $F(1,80)=5.55, p=0.02, R^{2}=0.07$.

\section{Mood and affect}

All groups showed comparable, and low $(M=9.97)$, current levels of depression as assessed by BDI-II scores, $F(2,86)=0.90$, $p=0.4$. In addition, all groups showed similar negative affect at baseline, $F(2,86)=1.29, p=0.3$. Although participants reported similar levels of positive affect at baseline across groups, 
$F(2,86)=2.24, p=0.12$, a marginal difference emerged between the CSt $(M=27.10)$ and the USt $(M=29.96)$ groups indicating that people randomly assigned to uncontrollable stress also had higher positive affect at baseline, $F(1,86)=2.98, p=0.09$, $R^{2}=0.03$. Including baseline positive affect in subsequent analyses, however, failed to alter any results; therefore we report simple analyses only. Finally, there were no differences between groups in change in negative, $F(2,86)=1.66, p=0.2$, or positive, $F(2,86)=0.18, p=0.8$, affect over the course of testing.

\section{DISCUSSION}

These results support the view that non-contingency is an active ingredient in the effects of uncontrollability on the relationship between subjective stress and executive functioning. Once again, stress response only predicted Stroop interference in the group exposed to controllable (contingent) stressors.

\section{GENERAL DISCUSSION}

Stress is ubiquitous, and previous research suggests it can have both negative (Oei et al., 2006; Schoofs et al., 2008, 2009) and positive (Duncko et al., 2009; Weerda et al., 2010) effects on cognitive function. However, from this previous research it was not clear what determines whether stress enhances or impairs function. The goal of the current studies was to investigate two important factors that may moderate the effect of stress on executive functioning: controllability of stress, and individual differences in subjective response to stress. Our results suggest that controllability and subjective response interact to determine whether stress exposure will impair or enhance Stroop performance: exposure to controllable stress that was experienced as moderately intense predicted improved performance (reduced interference), but subjective stress was unrelated to performance when stress exposure was uncontrollable. In addition, people exposed to controllable stress showed greater improvement in accuracy on incongruent trials than people exposed to uncontrollable stress, a result that further supports the benefits of behavioral control.

These results are consistent with previous research suggesting the importance of controllability (Dickerson and Kemeny, 2004; Arnsten, 2009) and intensity (Lupien et al., 1999, 2007) in moderating the effects of stress exposure on behavioral functioning. Importantly, our findings extend this literature to examine how these dimensions interact. In addition, this research uses experimental manipulations of controllability together with assessment of individual differences in subjective stress response to permit detection of linear and quadratic effects of stress on EF. In Experiment 1 , there was a quadratic relationship between subjective stress and Stroop performance across the full sample, and this effect was strongest within the group exposed to controllable stress. In Experiment 2, a similar quadratic relationship was detected across the full sample, but the group exposed to controllable stress showed a linear effect of subjective stress on Stroop performance. Because the dosage of controllable stress (proportion of trials that were failure/long noise) was higher in Experiment 2, this linear effect could reflect the fact that this group was shifted higher on the U-shaped subjective stress curve than similar participants in Experiment 1, and our analyses thus captured the upward slope of this quadratic relationship. The average subjective response to controllable stress in Experiment 1 was 0.50 standard deviations above the response reported by the no-stress group, but in Experiment 2 the response to controllable stress was 0.85 standard deviations above that reported by the no-stress group. Together, these results support the hypothesis that exposure to moderate and controllable stress causes improved functioning, while exposure to (subjectively) more intense or uncontrollable stress impairs functioning.

In addition, these results demonstrate for the first time that controllability and subjective stress influence the effect of stress on a measure of general EF (i.e., common EF; Friedman et al., 2008), providing more precise evidence for the specific cognitive systems affected by these factors. Previous research investigating the effects of stress on cognitive functioning has largely focused on declarative or working memory tasks, but prefrontal cortical systems that are sensitive to stress and undergird aspects of working memory also support other types of EFs. For example, EFs include the ability to hold goals in mind and resist interference from distractors. To investigate the effects of stress on common EF processes in this research, the color-word Stroop was used because it has been shown to load strongly on a common EF factor (Friedman et al., 2008). We would expect that performance on the Stroop would improve with practice (Logan, 1988) and indeed participants who were not exposed to stress showed improved performance over testing. However, the predictive relationship between subjective stress and Stroop performance emerged only for subjects exposed to controllable stress. These results support the hypothesis that controllability and subjective stress moderate the effect of stress on core cognitive abilities that are recruited not only for working memory tasks, but also for other tasks that require EFs.

Comparing Experiments 1 and 2 leads to some tentative conclusions regarding the critical aspects of controllability that affect behavioral functioning. Specifically, these results suggest that noncontingency between behavioral responses and stressors is sufficient to alter the relationship between subjective stress and Stroop performance, as demonstrated by Experiment 2. These results are consistent with the theory that exposure to non-contingency causes disruption to basic learning systems (Oakes, 1982; Oakes and Curtis, 1982).

The results of these experiments raise several questions. First, the current study is limited by the absence of physiological measures of stress reactivity, which would enable us to compare subjective and objective individual differences in stress responses. Previous research has shown that subjective ratings are related to physiological reactions, but are not perfectly correlated (Elzinga and Roelofs, 2005; Alexander et al., 2007; Schoofs et al., 2009). Our stress manipulation may have evoked responses in key stress systems, e.g., the hypothalamic-pituitary-adrenal (HPA) axis and sympathetic nervous system (SNS), but it is not possible to determine the degree or timing of such responses without physiological measures. Assessing multiple indices of physiological stress response would provide insight on the correspondence between subjective and objective reactivity to controllable or uncontrollable stress exposure. In addition, such biological measures would help improve understanding of how controllable 
versus uncontrollable stress may affect the complex balance of activation within and between stress systems, and interactions between stress mediators (e.g., cortisol, catecholamines) as they affect cognition. Previous work has suggested that the effect of stress on cognitive functioning depends on the relative levels of cortisol and adrenergic activity, which are related but not perfectly correlated (Okuda et al., 2004; Elzinga and Roelofs, 2005; Roozendaal et al., 2006). Furthermore, one study suggested that controllable stress leads to greater adrenergic activity, while uncontrollable stress leads to greater cortisol response (Peters et al., 1998). Exploring how controllability affects relative recruitment of stress systems may provide insight on why controllability moderates the relationship between subjective stress and cognitive functioning.

Second, it remains an open question why subjective response to stress was related to Stroop performance in the group exposed to controllable stress, but not in the group exposed to uncontrollable stress. One possibility is that uncontrollable stress leads to different neural responses than controllable stress, and thus does not affect EF performance in the same way. In rodents, the inability to learn to escape a stressor is related to decreased activity in neural systems responsible for down-regulating activity in stress-response regions; thus stress responses are permitted to occur unchecked, ultimately leading to a sensitized arousal system that reacts more readily to mild provocation (Maier and Watkins, 2005). In humans, similar regulatory brain systems are involved in tuning down arousal and affect, and research on emotion regulation strategies has demonstrated that these systems are recruited in the service of purposeful regulation of emotion (Delgado et al., 2008). It may be that in humans, the presence of contingency associations along with moderately intensive stress enables recruitment of prefrontal brain systems that regulate arousal in a top-down manner. This active and successful top-down regulation may extend beyond regulation of arousal to a more general enhancement of top-down control processes, and hence improved EF. However, if an individual experiences those stressors as extreme, top-down regulation may be unsuccessful and therefore yield no benefit. Meanwhile, the absence of contingency for people exposed to uncontrollable stress could remove this source of top-down

\section{REFERENCES}

Alexander, J. K., Hillier, A., Smith, R. M., Tivarus, M. E., and Beversdorf, D. Q. (2007). Beta-adrenergic modulation of cognitive flexibility during success. J. Cogn. Neurosci. 19, 468-478.

Arnsten, A. F. T. (2009). Stress signaling pathways that impair prefrontal cortex structure and function. Nat. Rev. Neurosci. 10, 410-422.

Arnsten, A. F. T., and Goldman-Rakic, P. S. (1998). Noise stress impairs prefrontal cortical cognitive function in monkeys. Arch. Gen. Psychiatry 55, 362-368.

Beck, A. T., Steer, R. A., Ball, R., and Ranieri, W. F. (1996). Comparison of Beck depression inventories-IA and

regulation, so that the biological stress response of these people is more related to individual differences in bottom-up reactivity and thus does not predict the ability to recruit top-down control in a subsequent EF task. However, note that this explanation fails to explain the poorer Stroop performance on average across the uncontrollable stress group in Experiment 1 (which, however, did not replicate in Experiment 2). Research incorporating neurobiological methods in humans is thus needed to test this theory.

Finally, in these experiments we detected no effects of mood or affect. Because this research was conducted with a non-clinical sample, and the majority of participants (71\%) reported scores below the cut-point for mild dysphoria (BDI > 12; Kendall et al., 1987; Beck et al., 1996), the absence of statistically significant depression effects may be unsurprising. The absence of stress effects on affect may be due to the delay between exposure and administration of the state-affect measure; any mood effects of stress, or controllability, may have tapered while participants completed the Stroop task. Use of a self-report measure that queries about emotions experienced during the stress manipulation may provide a more sensitive assessment of affect (as in the subjective ratings of stress, which were retrospective). Alternately, it may be that the absence of group differences in affective change accurately reflects the absence of controllability or exposure effects on emotion in these experiments. As has been pointed out by previous researchers (e.g., Lupien et al., 2007), stress and emotion are not isomorphic; clarifying the relationships between stress responses (both subjective and objective), controllability, and emotion, remains an important target of clinical research.

The results of this research are relevant for anyone who wants to capitalize on the potential of stress to enhance goal-directed behavior, while minimizing the negative effects of stress. In particular, this research is relevant as we set physical or academic goals for ourselves that are tuned to our individual appraisals of difficulty and controllability. Future studies investigating controllability and reactivity in settings such as education or clinical treatment may provide insight on how stress exposure can be a powerful source of benefit for students, clients, and others hoping to reap the helpful effects of stress.

functioning. Hum. Brain Mapp. 25, 409-423.

Delgado, M. R., Nearing, K. E., LeDoux, J. E., and Phelps, E. A. (2008). Neural circuitry underlying the regulation of conditioned fear and its relation to extinction. Neuron 59, 829-838.

DeVellis, R. F., McEvoy-DeVellis, B., and McCauley, C. (1978). Vicarious acquisition of learned helplessness. J. Pers. Soc. Psychol. 36, 894-899.

Dickerson, S. S., and Kemeny, M. E. (2004). Acute stressors and cortisol responses: a theoretical integration and synthesis of laboratory research. Psychol. Bull. 130, 355-391.

Duncko, R., Johnson, L., Merikangas, K., and Grillon, C. (2009). Working memory performance after acute exposure to the cold pressor stress in healthy volunteers. Neurobiol. Learn. Mem. 91, 377-381.

Eisenberger, R., Leonard, J. M., Carlson, J., and Park, D. C. (1979). Transfer effects of contingent and noncontingent positive reinforcement: mechanisms and generality. Am. J. Psychol. 92, 525-535.

Eisenberger, R., Park, D. C., and Frank, M. (1976). Learned industriousness and social reinforcement. J. Pers. Soc. Psychol. 33, 227-232.

Elzinga, B. M., and Roelofs, K. (2005). Cortisol-induced impairments of working memory require acute sympathetic activation. Behav. Neurosci. 119, 98-103. 
Friedman, N. P., Miyake, A., Young, S. E., DeFries, J. C., Corley, R. P., and Hewitt, J. K. (2008). Individual differences in executive functions are almost entirely genetic in origin. J. Exp. Psychol. Gen. 137, 201-225.

Gernigon, C., Fleurance, P., and Reine, B. (2000). Effects of uncontrollability and failure on the development of learned helplessness in perceptualmotor tasks. Res. Q. Exerc. Sport 71, 44-54.

Hains, A. B., and Arnsten, A. F. T. (2008). Molecular mechanisms of stress-induced prefrontal cortical impairment: implications for mental illness. Learn. Mem. 15, 551-564.

Hiroto, D. S. (1974). Locus of control and learned helplessness. J. Exp. Psychol. 102, 187-193.

Hiroto, D. S., and Seligman, M. E. P. (1975). Generality of learned helplessness in man. J. Pers. Soc. Psychol. 31, 311-327.

Hirt, M., and Genshaft, J. (1981). Immunization and reversibility of cognitive deficits due to learned helplessness. Pers. Individ. Dif. 2, 191-196.

Jones, S. L., Nation, J. R., and Massad, P. (1977). Immunization against learned helplessness in man. J. Abnorm. Psychol. 86, 75-83.

Kendall, P. C., Hollon, S. D., Beck, A. T., Hammen, C. L., and Ingram, R. E. (1987). Issues and recommendations regarding use of the Beck Depression Inventory. Cogn. Ther. Res. 11, 289-299.

Kilpatrick-Tabak, B., and Roth, S. (1978). An attempt to reverse performance deficits associated with depression and experimentally induced helplessness. J. Abnorm. Psychol. 37, 141-154.

Klein, D. C., Fencil-Morse, E., and Seligman, M. E. P. (1976). Learned helplessness, depression and the attribution of failure. J. Pers. Soc. Psychol. 33, 508-516.

Klein, D. C., and Seligman, M. E. P. (1976). Reversal of performance deficits and perceptual deficits in learned helplessness and depression. J. Abnorm. Psychol. 85, 11-26.

Kofta, M., and Sedek, G. (1989). Repeated failure: a source of helplessness or a factor irrelevant to its emergence? J. Exp. Psychol. Gen. 118, 3-12.

Koller, P. S., and Kaplan, R. M. (1978). A two-process theory of learned helplessness. J. Pers. Soc. Psychol. 36, 1177-1183.
Kuhlmann, S., Piel, M., and Wolf, O. T. (2005). Impaired memory retrieval after psychosocial stress in healthy young men. J. Neurosci. 25 , 2977-2982.

Lansbergen, M. M., Kenemans, J. L., and van Engeland, H. (2007). Stroop interference and attentiondeficit/hyperactivity disorder: a review and meta-analysis. Neuropsychology 21, 251-262.

Lewis, R. S., Nikolova, A., Chang, D. J., and Weekes, N. Y. (2008). Examination stress and components of working memory. Stress 11, 108-114.

Logan, G. (1988). Towards an instance theory of automatization. Psychol. Rev. 95, 492-527.

Luethi, M., Meier, B., and Sandi, C. (2009). Stress effects on working memory, explicit memory, and implicit memory for neutral and emotional stimuli in healthy men. Front. Behav. Neurosci. 2, 1-9.

Lupien, S. J., Gillin, C. J., and Hauger, R. L. (1999). Working memory is more sensitive than declarative memory to the acute effects of corticosteroids: a dose-response study in humans. Behav. Neurosci. 113, 420-430.

Lupien, S. J., Maheu, F., Tu, M., Fiocco, A., and Schramek, T. E. (2007). The effects of stress and stress hormones on human cognition: implications for the field of brain and cognition. Brain Cogn. 65, 209-237.

MacLeod, C. (1992). The Stroop task: the "gold standard" of attentional measures. J. Exp. Psychol. Gen. 121, 12-14.

Maier, S. F. (1984). Learned helplessness and animal models of depression. Prog. Neuropsychopharmacol. Biol. Psychiatry 8, 435-446.

Maier, S. F., and Seligman, M. E. P. (1976). Learned helplessness: theory and evidence. J. Exp. Psychol. Gen. 105, 3-46.

Maier, S. F., and Watkins, L. R. (2005). Stressor controllability and learned helplessness: the roles of the dorsal raphe nucleus, serotonin, and corticotropin-releasing factor. Neurosci. Biobehav. Rev. 29, 829-841.

Maier, S. F., and Watkins, L. R. (2010). Role of the medial prefrontal cortex in coping and resilience. Brain Res. 1355, 52-60.

Matute, H. (1994). Learned helplessness and superstitious behavior as opposite effects of uncontrollable reinforcement in humans. Learn. Motiv. 25, 216-232.

Matute, H. (1995). Human reactions to uncontrollable outcomes: further evidence for superstitions rather than helplessness. Q. J. Exp. Psychol. 48B, 142-157.

Miller, W. R., and Seligman, M. E. P. (1976). Depression and learned helplessness in man. Behav. Res. Ther. 14, 7-17.

Miyake, A., Friedman, N. P., Emerson, M. J., Witzki, A. H., Howerter, A., and Wager, T. D. (2000) The unity and diversity of executive functions and their contributions to complex "frontal lobe" tasks: a latent variable analysis. Cogn. Psychol. 41, 49-100.

Mizoguchi, K., Ishige, A., Takeda, S., Aburada, M., and Tabira, T. (2004). Endogenous glucocorticoids are essential for maintaining prefrontal cortical cognitive function. $J$. Neurosci. 24, 5492-5499.

Oakes, W. F. (1982). Learned helplessness and defensive strategies: a rejoinder. J. Pers. 50, 515-525.

Oakes, W. F., and Curtis, N. (1982). Learned helplessness: not dependent upon cognitions, attributions, or other such phenomenal experiences. J. Pers. 50, 387-408.

Oei, N. Y. L., Everaerd, W. T. A. M. Elzinga, B. M., Van Well, S., and Bermond, B. (2006). Psychosocial stress impairs working memory at high loads: an association with cortisol levels and memory retrieval. Stress 9, 133-141.

Oei, N. Y. L., Tollenaar, M. S., Spinhoven, P., and Elzinga, B. M. (2009). Hydrocortisone reduces emotional distracter interference in working memory. Psychoneuroendocrinology 34, 1284-1293.

Okuda, S., Roozendaal, B., and McGaugh, J. L. (2004). Glucocorticoid effects on object recognition memory require trainingassociated emotional arousal. Proc. Natl. Acad. Sci. U.S.A. 101, 853-858.

Ordaz, S., and Luna, B. (2012). Sex differences in physiological reactivity to acute psychosocial stress in adolescence. Psychoneuroendocrinology. doi:10.1016/j.psyneuen.2012. 01.002

Peters, M. L., Godaert, G. L. R., Ballieux, R. E., van Vliet, M., Willemsen, J. J., Sweep, F. C. G. J., and Heijnen, C. J. (1998). Cardiovascular and endocrine responses to experimental stress: effects of mental effort and controllability. Psychoneuroendocrinology 23, 1-17.

Pittman, N. L., and Pittman, T. S. (1979). Effects of amount of helplessness training and internalexternal locus of control on mood and performance. J. Pers. Soc. Psychol. 37, 39-47.

Porcelli, A. J., Cruz, D., Wenberg, K., Patterson, M. D., Biswal, B. B., and Rypma, B. (2008). The effects of acute stress on human prefrontal working memory systems. Physiol. Behav. 95, 282-289.

Price, K. P., Tryon, W. W., and Raps, C. S. (1978). Learned helplessness and depression in a clinical population: a test of two behavioral hypotheses. J. Abnorm. Psychol. 87, 113-121.

Qin, S., Hermans, E. J., van Marle, H. J. F., Luo, J., and Fernandez, G. (2009). Acute psychological stress reduces working memory-related activity in the dorsolateral prefrontal cortex. Biol. Psychiatry 66, 25-32.

Roozendaal, B., Okuda, S., Van der Zee, E. A., and McGaugh, J. L. (2006). Glucocorticoid enhancement of memory requires arousalinduced noradrenergic activation in the basolateral amygdala. Proc. Natl. Acad. Sci. U.S.A. 103, 6741-6746.

Schoofs, D., Preuss, D., and Wolf, O. T. (2008). Psychosocial stress induces working memory impairments in an n-back paradigm. Psychoneuroendocrinology 33, 643-653.

Schoofs, D., Wolf, O. T., and Smeets, T. (2009). Cold pressor stress impairs performance on working memory tasks requiring executive functions in healthy young men. Behav. Neurosci. 123, 1066-1075.

Seligman, M. E. P. (1972). Learned helplessness. Annu. Rev. Med. 23, 407-412.

Sliwinski, M., Smyth, J. M., Hofer, S. M., and Stawski, R. S. (2006). Intraindividual coupling of daily stress and cognition. Psychol. Aging 21, 545-557.

Stroop, J. R. (1935). Studies of interference in serial verbal reactions. J. Exp. Psychol. 18, 643-662.

Tennen, H. (1982). A re-view of cognitive mediators in learned helplessness. J. Pers. 50, 526-541.

Tennen, H., Drum, P. E., Gillen, R., and Stanton, A. (1982a). Learned helplessness and the detection of contingency: a direct test. J. Pers. 50, 426-442.

Tennen, H., Gillen, R., and Drum, P. E. (1982b). The debilitating effect of exposure to noncontingent escape: a test of the learned helplessness model. J. Pers. 50, 409-425.

Thornton, J. W., and Jacobs, P. D. (1971). Learned helplessness in human subjects. J. Exp. Psychol. 87, 367-372. 
Thornton, J. W., and Powell, G. D. (1974). Immunization to and alleviation of learned helplessness in man. Am. J. Psychol. 87, 351-367.

Tomaka, J., Blascovich, J., Kelsey, R. M., and Leitten, S. L. (1993). Subjective, physiological, and behavioral effects of threat and challenge appraisal. J. Pers. Soc. Psychol. 65, 248-260.

Vallee-Tourangeau, F., Murphy, R. A., and Baker, A. G. (2005). Contiguity and the outcome density bias in action-outcome contingency judgments. Q. J. Exp. Psychol. 58B, 177-192.

Watson, D., and Clark, L. A. (1999). The PANAS-X: Manual for the Positive and Negative Affect Schedule - Expanded Form, 2nd Edn. Iowa: University of Iowa.

Weerda, R., Muehlhan, M., Wolf, O. T., and Thiel, C. M. (2010). Effects of acute psychological stress on working memory-related brain activity in men. Hum. Brain Mapp. 31, 1418-1429.
Conflict of Interest Statement: The authors declare that the research was conducted in the absence of any commercial or financial relationships that could be construed as a potential conflict of interest.

Received: 15 March 2012; accepted: 16 May 2012; published online: 07 June 2012.

Citation: Henderson RK, Snyder HR, Gupta T and Banich MT (2012) When does stress help or harm? The effects of stress controllability and subjective stress response on Stroop performance. Front. Psychology 3:179. doi: 10.3389/fpsyg.2012.00179

This article was submitted to Frontiers in Emotion Science, a specialty of Frontiers in Psychology.

Copyright (c) 2012 Henderson, Snyder, Gupta and Banich. This is an open-access article distributed under the terms of the Creative Commons Attribution Non Commercial License, which permits noncommercial use, distribution, and reproduction in other forums, provided the original authors and source are credited. 